\title{
Editorial: Special Issue on "Information Security and Data Protection in Future Generation Communication and Networking"
}

\author{
Jong Hyuk Park • Han-Chieh Chao • Whai-En Chen • \\ Paris Kitsos
}

Published online: 10 October 2008

C) Springer Science+Business Media, LLC. 2008

The Future Generation Communication and Networking (FGCN) with advanced communication and networking environment is emerging for user centric consideration. In addition, the FGCN has emerged rapidly as an exciting new paradigm that includes ubiquitous, grid, and peer-to-peer computing to provide computing and communication services anytime and anywhere. Therefore, it is aim to provide users with more convenient and pleasurable services. While attack methods and tools have become more and more powerful, interest has greatly increased in the field of building, active and smart defense systems. Finding effective ways to protect information and data of FGCN environment is also a very challenging research problem.

This Call for Papers attracted 32 submissions from Asia, Europe and the US covering a lot of topics in the field of the FGCN. Each paper was carefully evaluated by three reviewers. This careful evaluation process has allowed us to select 15 high quality research papers.

Our special thanks go to Professor Ramjee Prasad (Editor-in-Chief) for his valuable support throughout the preparation of this Special Issue. We would like to thank all authors who have submitted papers to the Special Issue and in particular those whose papers have been accepted for this Special issue. Assistance from the editorial staff of the Wireless Personal Communications is also much appreciated. Finally, the Guest Editors wish to gratefully acknowledge all those who have generously given their time to review the papers submitted for consideration for this SI.

The fifteen accepted papers are divided into three categories. Five papers relate to Wireless or Vehicular Networks. "A Scalable Information Security Technique: Joint AuthenticationCoding Mechanism for Multimedia over Heterogeneous Wireless Networks" by Liang Zhou et al. discusses joint authentication coding for multimedia over wireless networks. "Improving WTLS Security for WAP based Mobile e-Commerce" by Yi-Jun He et al. tries to improve WTLS Security for WAP. "On Analyzing the Potential of a Layer-2 Multi-hop Authentication and Credential Delivery Scheme for Vehicular Communications" by Christian Tchepnda

J. H. Park $(\bowtie) \cdot$ H.-C. Chao · W.-E. Chen · P. Kitsos

Department of Computer Science and Engineering, Kyungnam University, 449 Wobyoung-dong,

Masan, Kyungnam, Korea

e-mail: parkjonghyuk1@ @otmail.com 
et al. analyzes the potential layer-2 multi-hop authentication and credential Delivery Scheme for vehicular networks. "Privacy Protected Spatial Query Processing for Advanced Location Based Services" by Wei-Shinn Ku et al. provides network distance spatial query solutions which protects user privacy by using K-anonymity mechanisms. "A new modulation for intrinsically secure radio channel in wireless systems" by Lorenzo Mucchi et al. proposes a new modulation for intrinsically secure radio channel in wireless systems.

Three papers are relative to Mobile Communications. "Fair and Secure Mobile Billing Systems" by Shiqun Li et al. proposes a fair and secure billing system for mobile communications. "Reducing Signaling Traffic for the Authentication and Key Agreement Procedure in an IP Multimedia Subsystem" by Chung-Ming Huang et al. proposes an effective authentication and key arrangement procedure in IMS. "Supporting Adaptive Context-Aware Services and Novel Handover Scheme for Smart Ubiquitous Communication System" by Chin-Feng Lai et al. provides adaptive context-aware services and a novel handover scheme for smart ubiquitous communications.

The last seven papers are about Personal or P2P network. "Establishing Trust through Anonymous and Private Information Exchange over Personal Networks" by Charalampos Z. Patrikakis et al. establishes trust through anonymous and private information exchange. "Action Patterns Probing for Dynamic Service Composition in Home Network" by Ju-Hsien Chou et al. provides action pattern probing for dynamic service composition in home network. "TACS, a Trust Model for P2P Networks" by Felix Gomez Marmol et al. proposes a trust model for P2P networks. "Protecting Your Privacy with a Mobile Agent Device in RFID Environment" by Sang-Soo Yeo et al. provides RFID privacy protection with mobile agent devices. "Fault-tolerance Data Aggregation for Clustering Wireless Sensor Network" by Shu Qin Ren and Jong Sou Park proposes an efficient privacy-preserving data fusions as well as malicious data tolerance. "RHS-US: Real-time Hash-chain Scheme decreasing Pre-calculation Mechanism for Ubiquitous Services" by Deok Gyu Lee et al. proposes a hash-chain scheme to improve the pre-calculation mechanism for RFID systems. Finally, "Towards a Performance-based Resource Server Access Control in Future Grid Communication Environments" by Naixue Xiong et al. proposes an effective access control algorithm to protect the critical resource.

\section{Author Biographies}

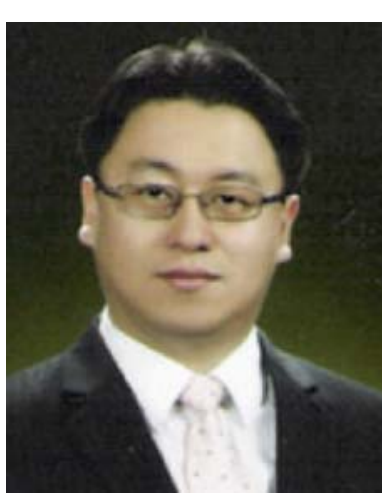

Jong Hyuk Park received his Ph.D. degree in Graduate School of Information Security from Korea University, Korea. Before August, 2007, Dr. Park had been a research scientist of R\&D Institute, Hanwha S\&C Co., Ltd., Korea. He is now a professor at the Department of Computer Science and Engineering, Kyungnam University, Korea. Dr. Park has published many research papers in international journals and conferences. Dr. Park has been served as Chairs, program committee or organizing committee chair for many international conferences, symposia and workshops. Dr. Park is the founder of International Conference on International Conference on Multimedia and Ubiquitous Engineering (MUE), International Conference on Intelligent Pervasive Computing (IPC), and International Symposium on Smart Home (SH). Dr. Park is editor-in-chief of the International Journal of Multimedia and Ubiquitous Engineering (IJMUE), the managing editor of the International Journal of Smart Home (IJSH), and Associate Editor of Security and Communication Networks ( $\mathrm{SCN}$ ). In addition, he has been served as a Guest Editor for international journals by some publishers: Oxford, Emerald, Hindawi, Springer, Elsevier, John Wiley, Inderscience, SERSC. Dr. Park has won a Best Paper Award of the 2nd International Conference on Information Security and Assurance (ISA 2008). Dr. Park's research interests include Digital Forensics, 
Security, Ubiquitous and Pervasive Computing, Context Awareness, Multimedia Service, etc. He is a member of the IEEE, IEEE Computer Society, IEEE Communications Society, KICS, KIISC, KMMS, KDFS and KIIT.

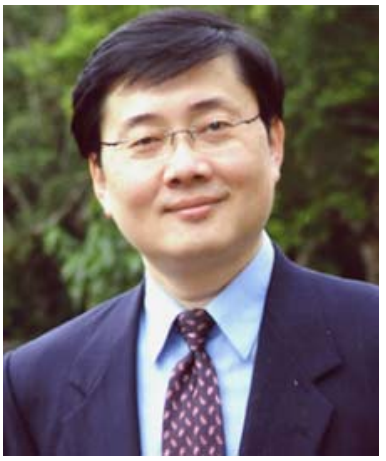

Han-Chieh Chao is a joint appointed Full Professor of the Department of Electronic Engineering and Institute of Computer Science \& Information Engineering at National Ilan University, Taiwan. He also serves as the Dean of the College of Electrical Engineering \& Computer Science and Director of Computer \& IT Center. At the same time, he holds the adjunct joint professorship with the Department of Electrical Engineering, National Dong Hwa University, Hualien, Taiwan. His research interests include High Speed Networks, Wireless Networks, IPv6 based Networks, Digital Creative Arts and Digital Divide. He received his MS and Ph.D. degrees in Electrical Engineering from Purdue University in 1989 and 1993, respectively. Dr. Chao has served as the guest editors for Mobile Networking and Applications (ACM MONET), IEEE JSAC, IEEE Communications Magazine, Computer Communications, IEE (IET) Proceedings Communications, Wireless Communications \& Mobile Computing, the Computer Journal, Wireless Personal Communications, Telecommunication Systems, Multimedia Tools and Applications, Simulation Modeling Practice and Theory and International Journal of Communications Systems. Dr. Chao is an IEEE senior member, a Fellow of the Institution of Engineering and Technology (FIET), and a Chartered Fellow of British Computer Society (FBCS).

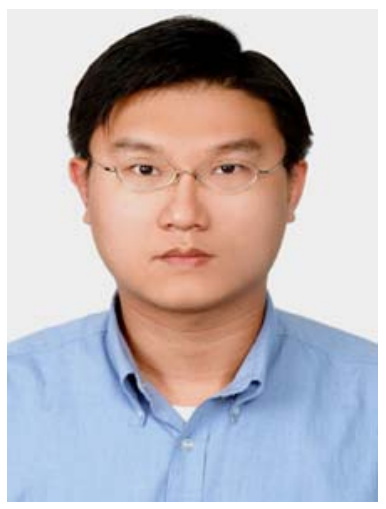

Whai-En Chen received a B.S. degree in electric engineering from Tam Kang University in 1997 and received a Ph.D. degree in computer science from National Tsing Hua University in 2002. He served as a research assistant professor at National Chiao Tung University from 2002 to 2007. Since August 2007 he is an assistant professor at the Institute of Computer Science and Information Engineering and chief of the network division of the computer and IT center of National Ilan University, I-Lan, Taiwan. His research interests include IP multimedia subsystem (IMS), SIP-based VoIP services, IPv6 translation mechanisms, Mobile IPv4/IPv6 and IP lookup/packet classification.

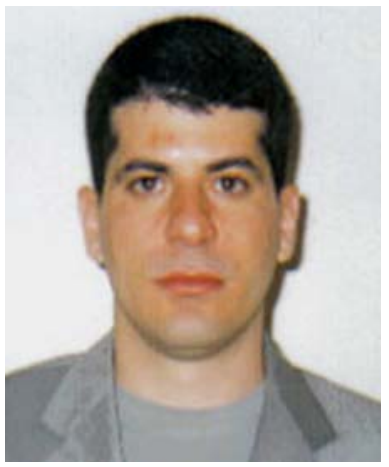

Paris Kitsos received the B.Sc. degree in Physics in 1999 and a Ph.D. in 2004 from the Department of Electrical and Computer Engineering, both at the University of Patras. Currently is research fellow with the Digital Systems \& Media Computing Laboratory, School of Science \& Technology, Hellenic Open University (HOU). His research interests include VLSI design, hardware implementations of cryptographic algorithms and security protocols for wireless communication systems. Dr. Kitsos has published more than 60 scientific articles and technical reports, as well as is reviewing manuscripts for International Journals and Conferences/Workshops in the areas of his research. He has participated to international journals and conferences organization, as Program/Technical Committee Member and Guest Editor. Also, is a member of the Institute of Electrical and Electronics Engineers (IEEE) and Institution of Engineering and Technology (IET). 